\title{
Surgery for acute proximal non-communicating aortic dissection without intimal tears (intramural hematoma)
}

\author{
Yutaka Okita \\ Takatsuki General Hospital, Kobe University, Chuo-ku, Kobe, Japan \\ Correspondence to: Yutaka Okita, MD, PhD. Takatsuki General Hospital, Kobe University, 7-5-2 Kusunoki-cho, Chuo-ku, Kobe 650-0017, Japan. \\ Email: yokita@med.kobe-u.ac.jp.
}

Submitted Apr 24, 2019. Accepted for publication Aug 02, 2019.

doi: 10.21037/acs.2019.08.09

View this article at: http://dx.doi.org/10.21037/acs.2019.08.09

\section{Introduction}

Our treatment strategy for Stanford type A noncommunicating acute aortic dissection is as follows. Emergency surgery should be considered for patients with complicated aortic valve insufficiency, cardiac tamponade, and continued pain. When there is ulcer-like projection (ULP) in the ascending aorta, surgery at an early stage should be considered. Other high risk features that may indicate urgent surgery, dependent on other patient factors, include: an aortic diameter of $>50 \mathrm{~mm}$, a hematoma thickness of $\geq 11 \mathrm{~mm}$, compression of the true lumen to $75 \%$ or less of the total aortic diameter, or delayed staining of contrast material into the false lumen. In cases not displaying these features, initial medical management seems to be appropriate, however, regular surveillance diagnostic imaging must be performed with an increase in the size of the thrombosed false lumen or a change to ULP or communicating type dissection indicating urgent surgery.

\section{Clinical vignette}

A 55-year-old male was diagnosed with an acute type A aortic dissection after complaining of severe back pain. The intimal tear was located in the descending aorta, and the false lumen of the ascending aorta was thrombosed. Initial medical therapy was started, but the diameter of the descending aorta was enlarged. A stent-graft was inserted in the descending aorta after one week. Ten days later, he complained of pain again, and a new entry tear was found on the distal arch. Chest computed tomography (CT) showed a thrombosed false lumen in the ascending aorta and the maximum diameter was $47 \mathrm{~mm}$ at the distal arch.
There was no aortic regurgitation or pericardial effusion. The dissection extended to both iliac arteries and all visceral arteries were branched from the true lumen. A separate aortic abdominal aneurysm was found.

\section{Pre-operative imaging study in the emergency room}

Transthoracic echocardiography is always performed to determine if there is a patent false lumen in the ascending aorta and to rule out any cardiac lesions, such as pericardial effusion, aortic valve regurgitation, left ventricular dysfunction and other valve function. CT aortogram with delayed phase images is mandatory to determine indications for surgery.

Standard general anesthesia is used, avoiding any episodes of hypertension. Regional oxygen saturation of the brain is measured with bitemporal near-infrared spectroscopy. Transesophageal echocardiography is always used after induction of anesthesia. Direct epiaortic ultrasound is used to detect any intimal tear or to determine an arterial cannulation site.

\section{Surgical technique}

\section{Position}

The patient is positioned supine and draped to provide access to the sternum, bilateral femoral vessels, axillary arteries, and the common carotid arteries. A skin incision is made from the sternal notch to the xiphoid process, and full mid-sternotomy is applied. A normal retractor is applied and the handle is placed on the right side of the surgeon. 
A longitudinal pericardiotomy is made, and extended as reversed a T-shape. If the patient has cardiac tamponade or a large pericardial effusion, the pericardium is not fully opened to prevent hypertension. Instead, an initial $1 \mathrm{~cm}$ pericardiotomy is performed to relieve the tamponade.

\section{Arterial or venous cannulation}

The ascending aorta is selected for arterial access after careful examination with epiaortic ultrasound. Usually, the false lumen is located on the left side of the ascending aorta, and direct cannulation using the Seldinger technique into the ascending aorta is appropriate. If there is thrombosis involving the full circumference of the ascending aorta, ascending aortic cannulation is contraindicated due to the risk of embolization. In these circumstances, peripheral arterial cannulation is employed using the axillary or femoral arteries.

Bicaval venous drainage is employed with a $36 \mathrm{Fr}$ straight cannula inserted from the right atrial appendage to the superior venous cava and a $28 \mathrm{Fr}$. angled cannula from the right atrium to the inferior vena cava. We routinely use retrograde cardioplegia delivered via a coronary sinus catheter. The left ventricle was vented using a right superior pulmonary vein catheter.

The patient was cooled for 15 to 20 minutes and reached $23{ }^{\circ} \mathrm{C}$ tympanic temperature and $30^{\circ} \mathrm{C}$ rectal temperature.

\section{Aortic clamp}

An aortic cross-clamp was applied to the ascending aorta in this particular case because the thrombi were not thick and there was a clear intimal tear in the descending aorta. However, cross-clamping is usually avoided to minimize the risk of embolism of false lumen hematoma through a previously unrecognized false lumen.

The ascending aorta was transected, in this case, fresh thrombus was found in the false lumen and there was no intimal tear. The aorta was trimmed at the level of the sinotubular junction (STJ), and the outer Teflon felt was fixed with 5-0 polypropylene sutures at three commissures. The false lumen was obliterated by an over and over continuous suture of 5-0 polypropylene suture. No biological glue was used.

\section{Brain protection}

After positioning the patient in a deep Trendelenburg position, systemic circulatory arrest was established. The aortic arch was opened, and three balloon-tipped cannulae were inserted from inside the aortic arch into the arch vessels. The regular size of the balloon is $15 \mathrm{Fr}$ for the brachiocephalic artery and $12 \mathrm{Fr}$ for the left common carotid (LCC) and left subclavian arteries (LSA). Great care is taken not to embolize debris when inserting the cannula. Brain perfusion flow is administered at $10-12 \mathrm{~mL} /$ body weight $(\mathrm{kg}) /$ minute and a separate roller pump is used for brain perfusion. The brain perfusion flow is controlled according to bilateral radial arterial pressure $(40-50 \mathrm{mmHg})$ and brain oxygen saturation $\left(\mathrm{SrO}_{2}\right)$.

\section{The intimal tear}

A large intimal tear was located $3 \mathrm{~cm}$ distal to the LSA on the dorsal site. The arch was dissected to the level of entry beyond the ligamentum arteriosus. A $20 \mathrm{~mm}$ diameter, 5 cm-length woven Dacron graft (Gelweave ${ }^{\circledR}$, Terumo, Glasgow) was inserted into the true lumen of the descending aorta as a free elephant trunk. No frozen elephant trunk (FET) was used in this particular case. The elephant trunk was fixed with an outer Teflon felt strip with three 4-0 polypropylene mattress sutures.

\section{Distal anastomosis}

Biological glue, such as Bioglue, GRF glue or fibrin glue, is never applied in the distal anastomosis. A $26 \mathrm{~mm}$ woven Dacron four-branch graft (Gelweave ${ }^{\circledR}$, Terumo, Glasgow) was anastomosed to the stump of the descending aorta with a continuous over and over technique using a 4-0 monofilament suture with $22 \mathrm{~mm}$ needle. Suturing was started at 9 o'clock with a forehand manner posteriorly and changed to a back-hand manner from 3 o'clock to 9 o'clock anteriorly. After distal anastomosis, antegrade perfusion of the distal lower body was done through the side branch of the graft, and systemic rewarming was started.

\section{Proximal anastomosis}

A continuous over and over suture of a 4-0 monofilament suture with $22 \mathrm{~mm}$ needle is used for proximal anastomosis, and then the heart was reperfused.

\section{Reconstruction of the arch vessels}

Three separate buttons of the arch vessels were made, and 
each button was anastomosed to the graft branch with a 5-0 polypropylene suture with a Teflon reinforcement. Usually, reconstruction of the LSA is done followed by the LCC artery finishing with the innominate artery, however, in this case the second the innominate artery anastomosis was completed second due to unstable right sided $\mathrm{SrO}_{2}$ readings. The rewarming was completed within 30 minutes and the patient was weaned from cardiopulmonary bypass (CPB). Postoperative CT scans showed a satisfactory result with complete thrombosis of the false lumen of the descending aorta.

\section{Results}

From October 1999 to March 2018, 359 patients with acute type A dissection underwent surgery for aorta and 38 patients had non-communicating aortic dissection without intimal tear (intramural hematoma). Mean age was $71 \pm 10$ years, and male:female ratio was $15: 23$. Surgery consisted of ascending aorta to hemiarch replacement in 20 patients and total arch replacement in 18 patients. There was no hospital mortality, and no new permanent neurological dysfunction was detected. In the same period, there were 18 patients with acute type A intramural hematoma (IMH) who had medical treatment as they did not meet surgical indications, with no mortality in this group.

\section{Discussion}

There is a discrepancy in the view as to the treatment strategy for Stanford type A non-communicating aortic dissection between Western countries and Japan or South Korea $(1,2)$. It is also common within the country for surgeons and internists to have different opinions on this issue. Kitai et al. (3) reported that a 10-year survival of 50 patients who had IMH type A and who had medical treatment was $89 \%$. Song et al. (4) reported a similar longterm survival of $83 \%$ in 85 patients with acute IMA type A who had medical treatment. Both of these reports do not neglect the importance of surgical treatment when the IMH meets surgical indication, and they underscore better freedom from death or morbidity in medical treatment than surgery. However, most of the papers from Western countries recommend early surgery for all patients who have acute IMH type A.

Surgery for acute IMH is rather simple, and replacement of the ascending aorta is the basic principle (5). However, when the initial intimal tear is located in the arch, we do not hesitate to replace the arch. Organ malperfusion secondary to acute dissection never happens in this entity, and standard CPB from the ascending aorta cannulation is usually used. Routine use of antegrade cerebral perfusion has provided satisfactory neurological outcomes. Application of the FET or the free elephant trunk in this subset of patients is still controversial. The FET may have stiffer lining within the true lumen but might cause stentinduced new entry. Surgical results are reported to be favorable, 30-day mortality was less than $5 \%$, and the new permanent or transient neurological deficit was also less than $5 \%$.

\section{Acknowledgments}

None.

\section{Footnote}

Conflicts of Interest: The author has no conflicts of interest to declare.

\section{References}

1. JCS Joint Working Group. Guidelines for diagnosis and treatment of aortic aneurysm and aortic dissection (JCS 2011): digest version. Circ J 2013;77:789-828.

2. Hiratzka LF, Bakris GL, Beckman JA, et al. 2010 ACCF/AHA/AATS/ACR/ASA/SCA/SCAI/SIR/STS/ SVM guidelines for the diagnosis and management of patients with Thoracic Aortic Disease: a report of the American College of Cardiology Foundation/ American Heart Association Task Force on Practice Guidelines, American Association for Thoracic Surgery, American College of Radiology, American Stroke Association, Society of Cardiovascular Anesthesiologists, Society for Cardiovascular Angiography and Interventions, Society of Interventional Radiology, Society of Thoracic Surgeons, and Society for Vascular Medicine. Circulation 2010;121:e266-369.

3. Kitai T, Kaji S, Yamamuro A, et al. Clinical outcomes of medical therapy and timely operation in initially diagnosed type a aortic intramural hematoma: a 20-year experience. Circulation 2009;120:S292-8. 
4. Song JK, Yim JH, Ahn JM, et al. Outcomes of patients with acute type a aortic intramural hematoma. Circulation 2009;120:2046-52.

5. Shimokawa T, Ozawa N, Takanashi S, et al. Intermediate-

Cite this article as: Okita Y. Surgery for acute proximal non-communicating aortic dissection without intimal tears (intramural hematoma). Ann Cardiothorac Surg 2019;8(5):570573. doi: $10.21037 /$ acs.2019.08.09 term results of surgical treatment of acute intramural hematoma involving the ascending aorta. Ann Thorac Surg 2008;85:982-6. 